\title{
Magellanic Clouds elemental abundances from F supergiants: Revisited results for the Large Magellanic Cloud ${ }^{\star}$
}

\author{
S. M. Andrievsky ${ }^{1,2}$, V. V. Kovtyukh ${ }^{1,2}$, S. A. Korotin ${ }^{1,2}$, M. Spite ${ }^{3}$, and F. Spite ${ }^{3}$ \\ 1 Department of Astronomy, Odessa State University, Shevchenko Park, 65014, Odessa, Ukraine \\ 2 Isaac Newton Institute of Chile, Odessa Branch \\ e-mail: serkor@skyline.od.ua \\ 3 Observatoire de Paris, DASGAL, UMR 8633 du CNRS, 92195 Meudon Cedex, France \\ e-mail: Monique.Spite@obspm.fr
}

Received 23 June 2000 / Accepted 30 November 2000

\begin{abstract}
With an improved method of the LTE abundance analysis for most elements (Kovtyukh \& Andrievsky 1999), the abundances have been re-investigated in nine F supergiants of the Large Magellanic Cloud. The NLTE calculations for carbon, oxygen and sodium, based on Kurucz's atmospheric models with an over-all metal deficiency, were carried out. The most important results are the following: - carbon abundance in supergiants is in much better agreement with the carbon abundance of the LMC H II regions, oxygen remaining in fair agreement with $\mathrm{H}$ II regions. The $(\mathrm{C} / \mathrm{O})$ ratio is low, lower than solar and the $\mathrm{H}$ II ratios (a possible sign of the first dredgeup, or another large-scale mixing event in the supergiant atmospheres); - the NLTE computations do not show remarkable sodium overabundances in the LMC supergiants, in contrast with the Galactic supergiants; - the mean abundance of iron is found to be $[\mathrm{Fe} / \mathrm{H}]=-0.40$, slightly lower than the results of some previous determinations for the LMC, but in agreement with the recent result obtained by Korn et al. (2000), who give $[\mathrm{Fe} / \mathrm{H}]=-0.41$. The relative to iron abundance of oxygen $[\mathrm{O} / \mathrm{Fe}]=+0.01$ derived from nine $\mathrm{F}$ supergiants appears to be in excellent accordance with predictions based on the smooth model of the LMC evolution; - the $(\alpha / \mathrm{Fe})$ ratios are comparable to those found in previous analyses. The surprisingly low abundance of $\mathrm{Mg}$ is even lower in the new analysis, but it is in good agreement with the relative magnesium abundance in LMC B-stars $[\mathrm{Mg} / \mathrm{H}]=-0.62$ determined by Korn et al. (2000). Sulphur in the program supergiants shows the same abundance within an error bar as in H II regions; - the iron-group elements follow the solar distribution of the $(\mathrm{M} / \mathrm{Fe})$ ratios; - the heavy elements show enhanced abundances; - significantly larger log $g$ values were obtained than in a previous study, resulting in supergiant masses of about $10-20 M_{\odot}$, which are in excellent agreement with evolutionary calculations.
\end{abstract}

Key words. stars: abundances - stars: NLTE -analysis - supergiants - Magellanic Clouds

\section{Introduction}

The chemical evolution of the Magellanic Clouds is the subject of many researches. Among the basic data, detailed stellar abundances are especially needed, but currently only the brightest stars are observable. The H II regions also provide an important information about abundances of the light elements in Magellanic Clouds. All these objects are young.

Recent spectroscopic analyses of $\mathrm{F}$ supergiants in the LMC have been performed by Hill et al. (1995) - hereafter HAS95, Luck \& Lambert (1992) - L L92, and Luck et al. (1998) - LET98. The latter paper gives a thorough discussion of the abundances in yellow supergiants obtained

Send offprint requests to: S. M. Andrievsky,

e-mail: scan@deneb.odessa.ua

* Based on spectra collected at ESO La Silla, Chile. by different authors during the last decade. In brief, the following points can be outlined:

Comparison to the Sun:

- The iron abundance determinations of the LMC supergiants, relative to the Sun, range from $[\mathrm{Fe} / \mathrm{H}]=$ $-0.36 \pm 0.22$ (LL92) to $-0.27 \pm 0.06$ (HAS95). We recall here the classical notation $[X]=\log \varepsilon(X)_{*}-\log \varepsilon(X)_{\odot}$;

- The carbon abundance in LMC supergiants is found to be between $[\mathrm{C} / \mathrm{H}]=-0.58 \pm 0.26-\mathrm{LL} 92$, and $-0.46 \pm 0.14-$ HAS95 (hereafter for the sake of uniformity the absolute $\mathrm{C}$ and $\mathrm{O}$ abundances given by different authors will be referred to the corresponding solar values $A(\mathrm{C}) \equiv \log \varepsilon(\mathrm{C})=8.55$ and $A(\mathrm{O}) \equiv$ $\log \varepsilon(\mathrm{O})=8.90$ adopted in this work and described in Sect. 3.3.4). The latter result is clearly larger than 
Table 1. Photometrical data and atmosphere parameters for program stars

\begin{tabular}{cccccccccc}
\hline Star & $E(B-V)$ & $V_{0}$ & $(V-R)_{0}$ & $T_{\text {eff }}(V-R)_{0}$ & $(V-I)_{0}$ & $T_{\text {eff }}(V-I)_{0}$ & $T_{\text {eff }}(\operatorname{adopt})$ & $\log g$ & $V_{\mathrm{t}}$ \\
\hline G244 & 0.08 & 11.20 & 0.11 & 7200 & 0.21 & 7400 & 7300 & 1.1 & 6.5 \\
G258 & 0.08 & 10.92 & 0.21 & 6500 & 0.41 & 6610 & 6550 & 1.0 & 6.5 \\
G274 & 0.11 & 11.88 & 0.22 & 6450 & 0.44 & 6500 & 6500 & 1.2 & 5.0 \\
G319 & 0.10 & - & - & - & - & - & $7500^{*}$ & 1.8 & 4.5 \\
G396 & 0.10 & 11.61 & 0.14 & 6950 & 0.26 & 7150 & 7050 & 1.1 & 6.0 \\
G406 & 0.15 & 11.87 & 0.19 & 6600 & 0.38 & 6700 & 6650 & 1.2 & 5.0 \\
G439 & 0.11 & 11.51 & 0.25 & 6250 & 0.45 & 6480 & 6350 & 1.0 & 6.0 \\
G501 & 0.22 & 11.96 & 0.23 & 6380 & 0.45 & 6480 & 6450 & 1.4 & 5.2 \\
G538 & 0.06 & 11.22 & 0.27 & 6140 & 0.53 & 6200 & 6180 & 0.8 & 6.5 \\
\hline
\end{tabular}

* For the hottest star of the sample, the effective temperature was adopted following the estimate made by HAS95.

several estimates of carbon content in LMC HiI regions: see Dufour et al. (1982), Kurt \& Dufour (1998 and references therein), who find a carbon abundance ranging from $[\mathrm{C} / \mathrm{H}]=-0.65 \pm 0.15$ to $-0.74 \pm 0.22$;

- The $[\mathrm{O} / \mathrm{H}]$ ratio for LMC supergiants is $-0.45 \pm 0.09$ (HAS95), or $-0.22 \pm 0.23$ (LL92). The former value is in close agreement with the oxygen abundance found for $\mathrm{H}$ II regions in $\mathrm{LMC}([\mathrm{O} / \mathrm{H}] \approx-0.50 \pm 0.09$, see Kurt \& Dufour 1998);

- The $(\mathrm{Na} / \mathrm{Fe})$ ratio is found only slightly enhanced in the LMC supergiants, relative to the solar ratio, contrary to the significant enhancement found in the Galactic supergiants;

- The $(\alpha$-elements/Fe) ratio is slightly enhanced relative to the solar ratio;

- No significant deviations from the solar $(\mathrm{M} / \mathrm{Fe})$ ratios were found for iron-group elements;

- The heavy metals in LMC supergiants are moderately enhanced relative to iron.

The same conclusions are reached for the Magellanic Cepheids (LET98). All these results are found from LTE analyses, and it is well known that, especially for low gravity stars, the use of the LTE assumption is questionable. However, the standard analysis is not completely satisfactory, in particular, it provides a systematically low gravity (too low according to the evolution theory).

It is clear that the main problem, in luminous and metal-poor stars, is the overionisation, but Spite et al. (1989) have evaluated the effect of overionisation in the abundance determination in the SMC supergiants, and estimated that the effects were moderate.

Recently, Thévenin \& Idiart (1999) showed that the Fe I lines (which are traditionally used as a tool for determination of the atmospheric parameters) are affected by significant NLTE effects, mainly due to overionisation, and the effect is therefore especially important for metal-poor stars.

Recently also, Gratton et al. (1999) confirmed that the Fe I lines can be significantly affected by NLTE effects: their Fig. 11 clearly shows that departure coefficients for some levels of the Fe I model progressively depend upon optical depth. Especially, in the atmospheres of rather warm and luminous stars (similar to our program supergiants), all the levels of Fe I model are underpopulated, when compared with the LTE case, while Fe II is not affected.

HAS95 made an attempt to correct for overionisation, and found that the microturbulent velocity and the gravity have then both to be increased: the effect on abundances is moderate, and is often nearly negligible for abundance ratios.

Kovtyukh \& Andrievsky (1999 - hereafter KA99), have shown that using Fe II lines for microturbulent velocity determination, a higher microtubulent velocity and a higher gravity are also obtained, in the analysis of Cepheids. Metal abundances are only slightly changed (even less the abundance ratios), but the abundances of $\mathrm{C}$ and $\mathrm{O}$ are changed enough for becoming close to the theoretical predictions.

This modification is usable only when a sufficient number of Fe II lines are measurable, but unfortunately, unblended Fe II lines, neither too weak nor too strong are not numerous. Taking advantage of the good quality of the available spectra, we decided in the present work to re-analyze the sample of F-type LMC supergiants studied previously by HAS95, in the way described by KA99. Moreover, the elements $\mathrm{C}, \mathrm{O}$ and $\mathrm{Na}$ are in addition analysed in more detail, using NLTE computations (Korotin et al. 1999) described in Sect. 3.2. The spectra of the program stars and their reduction are described in detail in HAS95.

\section{Atmospheric parameters of program stars}

We used the recent $T_{\text {eff- }}(V-R)$ and $T_{\text {eff- }}(V-I)$ calibrations of Castelli et al. (1997) to derive the effective temperatures for the program stars (the corresponding electronic tables can be found using the following address: cfaku5.harvard.edu). These calibrations are built with the aim of being independent of the metallicity effects. The individual colours and reddenings for our stars were selected from Grieve \& Madore (1986). The temperatures are given in Table 1 . The results on microturbulent velocity and gravity, determined as described by KA99 
Table 2. LTE elemental abundances for program stars

\begin{tabular}{|c|c|c|c|c|c|c|c|c|c|c|c|c|c|c|c|}
\hline & \multicolumn{3}{|c|}{ G244 } & \multicolumn{3}{|c|}{ G258 } & \multicolumn{3}{|c|}{ G274 } & \multicolumn{3}{|c|}{ G319 } & \multicolumn{3}{|c|}{ G396 } \\
\hline Ion & {$[\mathrm{El} / \mathrm{H}]$} & $\sigma$ & $N$ & {$[\mathrm{El} / \mathrm{H}]$} & $\bar{\sigma}$ & $N$ & {$[\mathrm{El} / \mathrm{H}]$} & $\sigma$ & $N$ & {$[\mathrm{El} / \mathrm{H}]$} & $\sigma$ & $N$ & {$[\mathrm{El} / \mathrm{H}]$} & $\sigma$ & $N$ \\
\hline Mg I & -1.01 & - & 1 & -0.54 & - & 1 & -0.60 & - & 1 & -1.08 & - & 1 & - & - & - \\
\hline $\mathrm{Al}$ & - & - & - & - & - & - & -0.18 & 0.01 & 2 & - & - & - & - & - & - \\
\hline Si I & - & - & - & -0.15 & 0.13 & 14 & -0.21 & 0.14 & 12 & -0.10 & 0.21 & 4 & -0.30 & 0.29 & 5 \\
\hline S I & - & - & - & -0.19 & 0.18 & 2 & -0.27 & 0.02 & 2 & -0.15 & - & 1 & -0.16 & - & 1 \\
\hline Ca I & -0.21 & 0.17 & 9 & -0.34 & 0.11 & 9 & -0.33 & 0.18 & 9 & -0.26 & 0.12 & 9 & -0.19 & 0.22 & 4 \\
\hline Sc II & -0.61 & 0.35 & 4 & -0.50 & 0.37 & 2 & -0.52 & 0.21 & 3 & -0.49 & 0.22 & 7 & -0.81 & 0.17 & 2 \\
\hline Ti I & - & - & - & - & - & - & - & - & - & - & - & - & - & - & - \\
\hline Ti II & -0.51 & - & 1 & -0.34 & - & 1 & -0.33 & - & 1 & -0.49 & 0.11 & 6 & -0.38 & - & 1 \\
\hline Cr II & -0.43 & 0.08 & 9 & -0.28 & 0.10 & 5 & -0.54 & 0.11 & 3 & -0.44 & 0.08 & 11 & - & - & - \\
\hline Mn I & - & - & - & -0.59 & 0.04 & 2 & -0.59 & 0.12 & 2 & - & - & - & - & - & - \\
\hline $\mathrm{Fe} I$ & -0.40 & 0.16 & 31 & -0.32 & 0.13 & 85 & -0.40 & 0.15 & 73 & -0.37 & 0.17 & 38 & -0.45 & 0.15 & 33 \\
\hline Fe II & -0.40 & 0.12 & 17 & -0.30 & 0.08 & 21 & -0.37 & 0.12 & 17 & -0.39 & 0.16 & 31 & -0.47 & 0.12 & 12 \\
\hline $\mathrm{Ni}$ I & - & - & - & -0.39 & 0.25 & 3 & -0.46 & 0.33 & 3 & - & - & - & -0.49 & - & 1 \\
\hline $\mathrm{Y}_{\mathrm{II}}$ & -0.79 & - & 1 & -0.20 & 0.23 & 4 & -0.30 & 0.35 & 3 & -0.46 & 0.10 & 3 & -0.04 & - & 1 \\
\hline Zr II & - & - & - & -0.06 & - & 1 & - & - & - & +0.06 & - & 1 & - & - & - \\
\hline Ba II & -0.07 & - & 1 & - & - & - & - & - & - & +0.06 & 0.11 & 2 & - & - & - \\
\hline La II & - & - & - & +0.15 & - & 1 & -0.01 & 0.34 & 2 & - & - & - & -0.07 & - & 1 \\
\hline Ce II & - & - & - & +0.00 & 0.01 & 2 & -0.25 & - & 1 & -0.14 & 0.15 & 2 & - & - & - \\
\hline Pr II & - & - & - & -0.07 & - & 1 & - & - & - & - & - & - & - & - & - \\
\hline $\mathrm{Nd}$ II & - & - & - & -0.15 & 0.15 & 5 & - & - & - & - & - & - & - & - & - \\
\hline \multirow[t]{2}{*}{ Eu II } & - & - & - & -0.01 & - & 1 & -0.24 & - & 1 & - & - & - & - & - & - \\
\hline & \multicolumn{3}{|c|}{ G406 } & \multicolumn{3}{|c|}{ G439 } & \multicolumn{3}{|c|}{ G501 } & \multicolumn{3}{|c|}{ G538 } & \multicolumn{3}{|c|}{ mean } \\
\hline Ion & {$[\mathrm{El} / \mathrm{H}]$} & $\sigma$ & $N$ & {$[\mathrm{El} / \mathrm{H}]$} & $\bar{\sigma}$ & $N$ & {$[\mathrm{El} / \mathrm{H}]$} & $\bar{\sigma}$ & $N$ & {$[\mathrm{El} / \mathrm{H}]$} & $\bar{\sigma}$ & $\bar{N}$ & $n$ & {$[\mathrm{El} / \mathrm{H}]$} & $\sigma$ \\
\hline$\overline{M g} \mathrm{I}$ & -0.53 & - & 1 & -0.63 & - & 1 & -0.60 & - & 1 & -0.62 & - & 1 & 8 & -0.70 & 0.21 \\
\hline $\mathrm{Al} \mathrm{I}$ & - & - & - & - & - & - & -0.23 & 0.01 & 2 & -0.27 & - & 1 & 3 & -0.22 & 0.05 \\
\hline Si I & -0.10 & 0.12 & 12 & -0.33 & 0.18 & 18 & -0.14 & 0.14 & 11 & -0.26 & 0.12 & 14 & 8 & -0.21 & 0.09 \\
\hline $\mathrm{S}_{\mathrm{I}}$ & -0.16 & 0.06 & 2 & -0.43 & 0.18 & 2 & -0.27 & 0.17 & 2 & -0.29 & 0.28 & 5 & 8 & -0.26 & 0.25 \\
\hline $\mathrm{Ca}$ I & -0.30 & 0.10 & 8 & -0.42 & 0.11 & 9 & -0.39 & 0.07 & 8 & -0.46 & 0.13 & 10 & 9 & -0.33 & 0.09 \\
\hline Sc II & -0.46 & 0.24 & 5 & -0.63 & 0.22 & 3 & - & - & - & -0.63 & 0.21 & 2 & 8 & -0.55 & 0.11 \\
\hline $\mathrm{Ti}$ I & - & - & - & -0.11 & - & 1 & - & - & - & -0.43 & - & 1 & 2 & -0.27 & - \\
\hline Ti II & -0.38 & - & 1 & -0.53 & - & 1 & - & - & - & -0.55 & - & 1 & 8 & -0.46 & 0.09 \\
\hline V II & - & - & - & - & - & - & - & - & - & -0.74 & - & 1 & - & - & - \\
\hline $\mathrm{Cr}_{\mathrm{I}}$ & -0.56 & - & 1 & -0.56 & - & 1 & -0.59 & - & 1 & -0.63 & 0.19 & 2 & - & - & - \\
\hline Cr II & -0.43 & 0.08 & 6 & -0.55 & 0.06 & 6 & -0.45 & 0.09 & 6 & -0.55 & 0.08 & 6 & 8 & -0.45 & 0.09 \\
\hline Mn I & -0.43 & 0.05 & 2 & -0.60 & 0.04 & 2 & -0.53 & 0.04 & 2 & -0.73 & 0.07 & 3 & 6 & -0.58 & 0.10 \\
\hline $\mathrm{Fe} \mathrm{I}$ & -0.37 & 0.14 & 83 & -0.44 & 0.13 & 74 & -0.29 & 0.12 & 80 & -0.52 & 0.16 & 112 & 9 & -0.40 & 0.12 \\
\hline Fe II & -0.34 & 0.07 & 20 & -0.45 & 0.08 & 15 & -0.29 & 0.12 & 17 & -0.49 & 0.10 & 15 & 9 & -0.39 & 0.07 \\
\hline Co I & - & - & - & - & - & - & - & - & - & -0.36 & - & 1 & - & - & - \\
\hline $\mathrm{Ni} I$ & -0.37 & 0.14 & 2 & -0.75 & 0.13 & 3 & -0.57 & 0.03 & 2 & -0.74 & 0.16 & 11 & 7 & -0.61 & 0.16 \\
\hline $\mathrm{Cu} \mathrm{I}$ & - & - & - & - & - & - & - & - & - & -0.40 & 0.58 & 2 & - & - & - \\
\hline Zn I & - & - & - & - & - & - & - & - & - & -0.17 & - & 1 & - & - & - \\
\hline $\mathrm{Y}_{\mathrm{II}}$ & -0.16 & - & 1 & -0.19 & - & 1 & -0.17 & - & 1 & -0.43 & 0.17 & 5 & 9 & -0.30 & 0.22 \\
\hline Zr II & - & - & - & - & - & - & - & - & - & -0.44 & 0.08 & 2 & 3 & -0.14 & 0.26 \\
\hline La II & +0.05 & 0.35 & 2 & -0.12 & 0.26 & 2 & -0.05 & 0.23 & 2 & +0.02 & - & 1 & 7 & +0.00 & 0.09 \\
\hline Ce II & -0.03 & 0.08 & 2 & -0.12 & 0.11 & 2 & +0.01 & - & 1 & -0.41 & 0.17 & 5 & 7 & -0.13 & 0.15 \\
\hline Pr II & - & - & - & - & - & - & - & - & - & -0.51 & 0.16 & 2 & - & - & - \\
\hline $\mathrm{Nd}$ II & +0.02 & - & 1 & -0.07 & 0.19 & 3 & +0.05 & - & 1 & -0.30 & 0.22 & 10 & 5 & -0.09 & 0.14 \\
\hline Eu II & -0.07 & - & 1 & +0.02 & - & 1 & - & - & - & -0.23 & 0.16 & 2 & 5 & -0.11 & 0.12 \\
\hline
\end{tabular}

* The last three columns give the number of stars contributing to the mean, the mean abundance of the sample and the standard deviation.

(non standard approach: use of Fe II lines), are also given in Table 1.

\section{Results}

\subsection{Improved LTE analysis}

The LTE analysis has been made using Kurucz's WIDTH9 code (1992) and the grid of atmosphere models of Castelli et al. (1997) with an over-all metal deficiency of -0.3 dex. The line oscillator strengths used are discussed and presented in KA99 (they are obtained by inverse solar analysis). The broadening values were selected from CD-ROM No. 23 of Kurucz \& Bell (1995). In Table 2 we give the results of the determination of elemental abundances (except for carbon, oxygen and sodium abundances, presented in the next section). In Fig. 1 we also show graphically the averaged abundances which will be discussed below.

\subsection{NLTE abundances of carbon, oxygen and sodium}

The NLTE abundances of $\mathrm{C}, \mathrm{O}$ and $\mathrm{Na}$ were found with the help of a modified version of the MULTI code (Carlsson 1986) described in Korotin et al. (1999) and Korotin et al. (1999). In such a modified version, in particular, additional opacity sources from ATLAS9 code (Kurucz 1992) were included. This was done in order to calculate the continuum opacity more precisely, and to take into account the absorption by a great number of spectral lines (especially within the region of the nearUV). It allows then to calculate more accurately the intensity distribution in the region 900-1500 A. In turn, this significantly affects the determination of the radiative rates of $b-f$ transitions. A simultaneous solution of the radiative transfer and statistical equilibrium equations has been performed in the approximation of complete frequency redistribution for all the lines. All the NLTE 


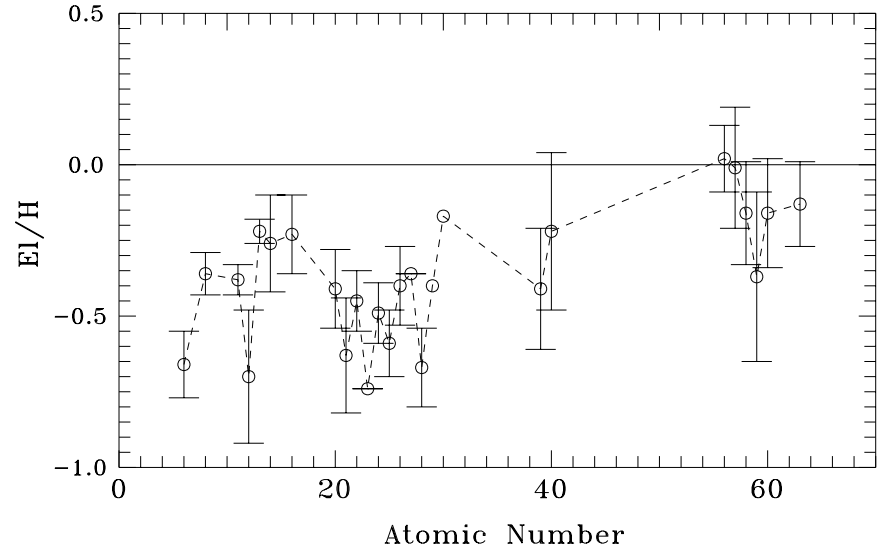

Fig. 1. Averaged elemental abundances. $2 \sigma$ intervals are indicated

calculations were also based on the Kurucz's grid of atmospheric models.

\subsection{Parameters of the considered atoms}

\subsubsection{Carbon}

We employed a model of carbon atom consisting of 115 levels: 94 levels of $\mathrm{C}$ I, 20 levels of $\mathrm{C}$ II and the ground state of $\mathrm{C}$ III. Within the described system of the carbon atomic levels, we considered the radiative transitions between the first 47 levels of C I and ground level of C II. Transitions between the remaining levels were not taken into account (they were used only in the equations of particle number conservation). After test calculations, 113 transitions were included in the linearization procedure. These transitions describe quite completely the formation of the lines of interest. The other 155 transitions were treated as those having fixed radiative rates.

\subsubsection{Oxygen}

In our computations, the oxygen atomic model consists of 75 levels: 72 levels of O I, 3 levels of O II and the ground state of O III (for details see Mishenina et al. 1999). The radiative transitions between the first 23 levels of $\mathrm{O}$ I and the ground level of O II were calculated.

\subsubsection{Sodium}

The model of sodium atom as described by Sahibullin (1987), has been modified (see Korotin \& Mishenina 1999). It consists of 27 levels of $\mathrm{Na}$ I and the ground level of Na II. We considered the radiative transitions between the first 20 levels of $\mathrm{NaI}$ and the ground level of $\mathrm{NaII}$. Transitions between the remaining levels were used only in the equations of particle number conservation. Finally, $46 b-b$ and $20 b-f$ transitions were included in the linearization procedure. For 34 transitions the radiative rates were fixed.
Table 3. Parameters of the selected lines

\begin{tabular}{cccccc}
\hline$\lambda$ & $g$ & $\log g f$ & $\lambda$ & $g$ & $\log g f$ \\
\hline $\mathrm{C}_{\mathrm{I}}$ & & \multicolumn{5}{c}{$\mathrm{Na \textrm {I }}$} \\
4775.91 & 5 & -2.27 & 5682.63 & 2 & -0.70 \\
5052.17 & 3 & -1.24 & 5688.21 & 4 & -0.46 \\
5380.34 & 3 & -1.57 & 5688.19 & 4 & -1.39 \\
6001.12 & 5 & -2.07 & 6154.23 & 2 & -1.53 \\
6010.68 & 3 & -2.02 & 6160.75 & 4 & -1.23 \\
6014.83 & 5 & -1.71 & & & \\
6413.55 & 3 & -2.38 & O I & & \\
6587.61 & 3 & -1.05 & 6155.98 & 3 & -0.67 \\
6671.85 & 5 & -1.66 & 6156.77 & 5 & -0.45 \\
7111.48 & 3 & -1.07 & 6158.18 & 7 & -0.31 \\
7113.18 & 7 & -0.76 & 6363.88 & 3 & -10.30 \\
7116.99 & 7 & -0.91 & 5577.34 & 5 & -8.24 \\
\hline
\end{tabular}

\subsubsection{General remarks}

A detailed structure of the multiplets was ignored in the calculations and each $L S$ multiplet was considered as a single term (with one exception for the $\mathrm{Na}$ I $3 \mathrm{p}^{2} \mathrm{P} 0$ level, whose structure has been taken into account in detail). After the combined solution of radiative transfer and statistical equilibrium equations, the averaged levels have been splitted with respect to multiplet structure. Then the level populations were redistributed proportionally to the statistical weights of the corresponding sublevels and finally the lines of interest were studied.

For all atomic models, the photoionization cross sections were mainly taken from the Opacity Project (Yan et al. 1987) keeping a detailed structure of their frequency dependence, including resonances. Collisional ionization was described using Seaton's formula (Seaton 1962). For all allowed $b-b$ transitions we used van Regemorter (1962) formula. Collisional rates for the forbidden transitions were calculated with the help of semiempirical formula (Allen 1973), with collisional force of 1 . As known, the elastic collisions with hydrogen atoms become rather significant in the atmospheres of cool stars. This effect has been taken into account with the help of Steenbock \& Holweger (1984) formula (with correcting factor 1/3).

For the NLTE calculation we used theoretical oscillator strengths from the catalogue by Hirata \& Horaguchi (1994), instead of the "solar" $\log g f$ determined under the LTE approximation from the solar spectrum. Some $\log g f$ for transitions from the ground level were selected from Verner et al. (1994) and from CD-ROM No. 23 by Kurucz \& Bell (1995). For C I the oscillator strengths from Biémont et al. (1993) were also used.

Van der Waals $C_{6}$ constant for the lines of considered elements was calculated using the classical formula of Unsöld. For the strong sodium lines the classical $C_{6}$ values were corrected by fitting the calculated and observed line wings in the solar spectrum (this procedure is described in Korotin \& Mishenina 1999). No correction factor to $C_{6}$ constant was used for the weak carbon and oxygen lines. In Table 3 we give the list of the carbon, 
Table 4. Averaged NLTE and LTE abundances of C, O and Na

\begin{tabular}{ccccccccccccc}
\hline & \multicolumn{2}{c}{ NLTE } & \multicolumn{2}{c}{ LTE } & \multicolumn{2}{c}{ NLTE } & \multicolumn{2}{c}{ LTE } & \multicolumn{2}{c}{ NLTE } & \multicolumn{2}{c}{ LTE } \\
\hline Star & $A(\mathrm{C})$ & $\sigma$ & $A(\mathrm{C})$ & $\sigma$ & $A(\mathrm{O})$ & $\sigma$ & $A(\mathrm{O})$ & $\sigma$ & $A(\mathrm{Na})$ & $\sigma$ & $A(\mathrm{Na})$ & $\sigma$ \\
\hline G244 & 7.92 & 0.06 & 8.05 & 0.05 & 8.43 & 0.11 & 8.46 & 0.12 & 5.98 & 0.06 & 6.07 & 0.08 \\
G258 & 7.95 & 0.07 & 8.01 & 0.07 & 8.68 & 0.04 & 8.70 & 0.05 & 6.03 & 0.01 & 6.12 & 0.04 \\
G274 & 7.80 & 0.19 & 7.85 & 0.14 & 8.50 & 0.07 & 8.50 & 0.09 & 5.82 & 0.04 & 5.94 & 0.05 \\
G319 & 7.86 & 0.15 & 7.86 & 0.19 & 8.47 & 0.03 & 8.47 & 0.06 & 5.79 & - & 5.87 & - \\
G396 & 7.90 & 0.03 & 7.93 & 0.07 & 8.50 & 0.12 & 8.52 & 0.11 & - & - & - & \\
G406 & 7.94 & 0.15 & 7.99 & 0.09 & 8.45 & 0.08 & 8.46 & 0.09 & 6.05 & 0.04 & 6.15 & 0.06 \\
G439 & 7.67 & 0.17 & 7.75 & 0.14 & 8.46 & 0.08 & 8.46 & 0.07 & 5.79 & 0.08 & 5.88 & 0.08 \\
G501 & 8.04 & 0.04 & 8.08 & 0.06 & 8.60 & 0.08 & 8.60 & 0.08 & 5.96 & 0.14 & 6.05 & 0.13 \\
G538 & 7.94 & 0.12 & 8.01 & 0.16 & 8.53 & 0.06 & 8.54 & 0.07 & 5.78 & 0.01 & 5.88 & 0.04 \\
\hline Mean & 7.89 & 0.11 & 7.95 & 0.11 & 8.51 & 0.08 & 8.52 & 0.08 & 5.90 & 0.12 & 6.01 & 0.11 \\
\hline Sun & 8.55 & 0.05 & & & 8.90 & 0.04 & & & 6.25 & 0.03 & & \\
\hline
\end{tabular}

oxygen and sodium lines and the oscillator strengths used for the NLTE calculations.

Table 4 contains the abundances of carbon, oxygen and sodium, averaged for each star. The abundances of the element $X$ are given as $A(X) \equiv \log \varepsilon(X)$. The mean abundance of $\mathrm{C}, \mathrm{O}$ and $\mathrm{Na}$ for the sample is provided in the bottom line, above the solar abundances. We also give for reference the computed solar NLTE abundances: they were determined from the lines listed in Table 3. Those lines were accurately measured from the solar flux spectrum (Kurucz et al. 1984). The abundances were calculated with Kurucz's solar atmosphere model. To take into account the chromospheric growth of the temperature, this model was completed by a model of the solar chromosphere from the work of Maltby et al. (1986). Nevertheless, the influence of a chromosphere on the calculated equivalent widths of the lines considered appeared to be insignificant (less than $2 \%$ ). Our calculated solar NLTE abundances of C, O and $\mathrm{Na}$, are in good agreement with the currently adopted abundances of these elements in the solar atmosphere. For example,

1) our solar carbon abundance, $A(\mathrm{C})=8.55$, is hardly different from the one given by Biémont et al. (1993) or Grevesse \& Sauval (1998): 8.57 and 8.52 respectively;

2) Anders \& Grevesse (1989), Grevesse \& Sauval (1998) and Biémont et al. (1991) derive $A(\mathrm{O})=8.93$, 8.83 and 8.86 respectively, while we find $A(\mathrm{O})=8.90$;

3) Our estimate of the sodium content $A(\mathrm{Na})=6.25$ agrees well with the determination of Takeda \& TakadaHidai (1994), who give $A(\mathrm{Na})=6.23$, while Grevesse \& Sauval (1998) give the meteoritic value $A(\mathrm{Na})=6.33$.

It has to be noted that, for our program $\mathrm{F}$ supergiants, the NLTE corrections lead to slightly lower abundances as compared to the LTE case. The differences between NLTE and LTE abundances are small (about of $0.10 \mathrm{dex}$ ) for carbon, oxygen and sodium. As discussed below, the deficiency of these elements, in the LMC supergiants, relative to the Sun, is therefore confirmed.
Table 5. Gravities and masses for program stars

\begin{tabular}{cccccc}
\hline & \multicolumn{3}{c}{ This paper } & \multicolumn{2}{c}{ HAS95 } \\
\hline Star & $M_{v}$ & $\log g$ & $M / M_{\odot}$ & $\log g$ & $M / M_{\odot}$ \\
\hline G 244 & -7.37 & 1.1 & 12.8 & 0.3 & 2.1 \\
G 258 & -7.65 & 1.0 & 20.3 & 0.1 & 2.6 \\
G 274 & -6.69 & 1.2 & 13.7 & 0.3 & 1.7 \\
G 319 & & 1.8 & & 1.0 & \\
G 396 & -6.96 & 1.1 & 10.1 & 0.6 & 3.5 \\
G 406 & -6.70 & 1.2 & 12.6 & 0.5 & 2.8 \\
G 439 & -7.06 & 1.0 & 13.3 & 0.4 & 3.2 \\
G 501 & -6.61 & 1.4 & 20.8 & 0.7 & 4.6 \\
G 538 & -7.35 & 0.8 & 12.2 & 0.1 & 2.4 \\
\hline
\end{tabular}

\section{Discussion}

\subsection{Model parameters}

In this work, some parameters of the supergiants are different from those found in HAS95. But the temperature values are only slightly different from those derived in HAS95. The difference never exceeds $200 \mathrm{~K}$, and is much smaller (about $50 \mathrm{~K}$ ) in the mean.

The abundance differences between both works have to be ascribed to differences in $V_{\mathrm{t}}, \log g$ and $\log g f$ and not only to temperature differences. The microturbulent velocities found by using the Fe II lines are from 1 to $3 \mathrm{~km} \mathrm{~s}^{-1}$ greater than those derived using the Fe I lines. The surface gravities for the program stars are significantly greater. In Table 5 we give the masses of program stars estimated using the usual expression:

$\log \left(M / M_{\odot}\right)=\log g-0.4\left(M_{\mathrm{bol}}-4.74\right)-4 \log T_{\mathrm{eff}}+10.616(1)$

for two different cases: spectroscopic gravities determined under the usual approach (HAS95), and using the method described by KA99.

The absolute visual magnitudes were found using the distance modulus for LMC $\left(V_{0}-M_{v}\right)=18.57$ (Sandage et al. 1999). We extrapolated the bolometric magnitude corrections for $\mathrm{F}$ supergiants of approximately zero from data of Castelli (1999). The gravities and masses found here are in agreement with the stellar evolution theory. This is clearly seen in Fig. 2. 


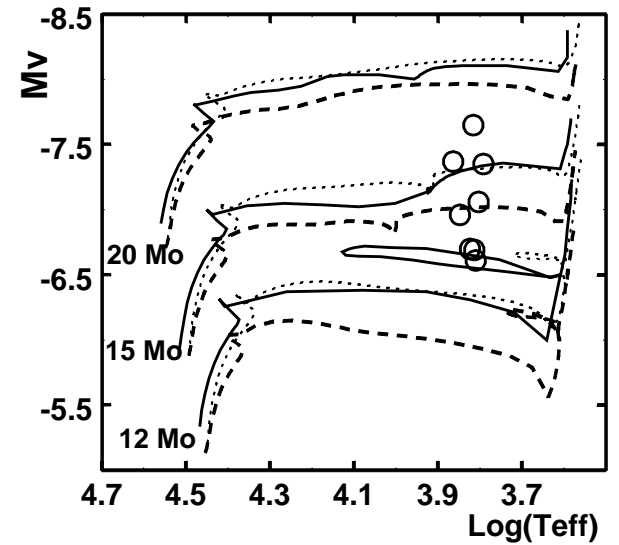

Fig. 2. Evolutionary tracks and position of program stars in HR diagram. Evolutionary tracks for $Z=0.01$ are from 1) Pols et al. (1998): standard models - dashed line, and models with overshooting - dotted line, 2) Schaller et al. (1992) - solid line

Table 6. Useful ratios for the light element abundances

\begin{tabular}{ccccc}
\hline Ratio & HAS95 & LL92 & H II & Our data \\
\hline$[\mathrm{C} / \mathrm{H}]$ & -0.46 & -0.58 & $-0.65 /-0.74$ & -0.66 \\
{$[\mathrm{O} / \mathrm{H}]$} & -0.45 & -0.22 & $-0.47 /-0.53$ & -0.39 \\
{$[\mathrm{Fe} / \mathrm{H}]$} & -0.27 & -0.36 & & -0.40 \\
{$[\mathrm{C} / \mathrm{Fe}]$} & -0.19 & -0.22 & & -0.26 \\
{$[\mathrm{O} / \mathrm{Fe}]$} & -0.18 & +0.14 & & +0.01 \\
$(\mathrm{C} / \mathrm{O})$ & -0.36 & -0.71 & $-0.53 /-0.56$ & -0.62 \\
\hline
\end{tabular}

The data for H II regions are from Dufour (1984), Dufour, Schields \& Talbot (1982) and Kurt \& Dufour (1998).

\subsection{Carbon and oxygen abundances}

Table 6 summarizes the useful ratios of carbon and oxygen abundances that will be used in the discussion below.

Previous estimate of carbon abundance in the sample of $\mathrm{F}$ supergiants (HAS95) gave $A(\mathrm{C})=8.10$. Our present NLTE mean result for the same sample of stars gives $A(\mathrm{C})=7.89$, in much better agreement with data on carbon abundance in LMC H II regions (7.90 and 7.81 accordingly to Dufour 1984 and Kurt \& Dufour 1998 respectively). However, the stellar carbon abundance may be expected to be lower than the HII abundance in case of dredge-up or partial mixing due to rotation, for example. A first dredge-up event in the phase of the red giant should produce the following effect: a surface carbon abundance becomes smaller (and nitrogen abundance larger, while oxygen remains practically unchanged) than that of $\mathrm{H}$ II regions, assumed to be made of the same young matter which formed the young supergiants. Of course H II regions may illuminate some less evolved matter, not yet enriched in carbon by intermediate mass stars (and this hypothesis has even been formalised by Pilyugin 1998), but the strikingly uniform metallicity of the LMC supergiants (HAS95, see also LET98) suggests on the contrary an efficient mixing of the young matter in the LMC. And a similar homogeneity has also been noted for H II regions (e.g., Pagel et al. 1978). In addition, it is commonly accepted that a rotating bar produces a considerable gas streaming in a galaxy, explaining this homogeneity.

One should also note that another source of the surface alteration of the carbon and nitrogen abundances in supergiant star may be identified with convection induced turbulence (i.e. overshooting into stable regions) during the previous main sequence evolution of the supergiant, when the star was the hot B dwarf. For example, Andrievsky et al. (1999) found among ten main sequence carbon deficient B stars three objects showing an increased nitrogen content.

Thus, the rather low ratio $(\mathrm{C} / \mathrm{O})$ found for our stars, which is lower than solar ratio $(\mathrm{C} / \mathrm{O})=-0.35$ and that of $\mathrm{H}$ II regions (see Table 6) seems to support the supposition about the first dredge-up (or turbulent mixing) in their history.

On the other hand, the position of the program stars in HR diagram with evolutionary tracks from Pols et al. (1998) seems to indicate that they are only on the first way towards the red giant region (see Fig. 2), because no blue loops are expected for the models of masses greater than $15 M_{\odot}$. At least for some program stars this conclusion remains valid with evolutionary tracks calculated by Schaller et al. (1992), while several of them occupy the position on blue loop (see Fig. 2), and an exact direction of their evolution within the HR is not clear.

There are only two possibilities to explain an actual position of our program stars in HR diagram: 1) either they (or some of them) are traveling to the red giant region for the first time, and their present atmospheric carbon abundance being unchanged is the same as that inherent to $\mathrm{HII}$ regions, or 2) the F supergiants may have already experienced the first dredge-up (or another kind of the large-scale mixing), and their initial carbon abundance was really decreased. In this case the rather low carbon abundance in H II (when compared with the supergiants' mean value) may result from the processes in which some part of the carbon and oxygen atoms are locked into grains in $\mathrm{H}$ II regions (owing to their low condensation temperature). Summarizing, we have also to state that based only on the measured carbon abundance and having not investigated the nitrogen content we cannot confidently conclude whether our program supergiants have already experienced the large-scale mixing, or not.

The oxygen abundance obtained for our sample of $\mathrm{F}$ supergiants is close to that of the interstellar medium in LMC, as expected, because the surface abundance of this element is not significantly changed by the supergiant evolution. The NLTE mean value of the oxygen abundance for our sample of $\mathrm{F}$ supergiants is $A(\mathrm{O})=8.51$ dex: it is higher than the previous LTE value (8.45 dex), but remains close to that of LMC HII regions (Dufour 1984; Kurt \& Dufour 1998 give 8.43 and 8.37 respectively). Our new value of oxygen abundance, referred to the mean iron abundance $(A(\mathrm{Fe})=7.10)$ and then to the corresponding solar ratio $(\mathrm{O} / \mathrm{Fe})_{\odot}$, gives $[\mathrm{O} / \mathrm{Fe}]=+0.01$. This ratio is slightly greater than zero, and anyway larger than previous estimate made by HAS95 (Table 6). The models of 
chemical evolution of the Magellanic Clouds (e.g., Pagel \& Tautvaisiene 1998) predict $[\mathrm{O} / \mathrm{Fe}]$ values near zero for continuous formation. This prediction from the bursting and smooth models for the metallicity $[\mathrm{Fe} / \mathrm{H}]$ of about -0.40 dex appears to be in the excellent agreement with our results, especially for the case of smooth evolution (see Fig. 6 from that paper). To reach an agreement between the theory predictions and observational data on the oxygen abundance derived in several previous studies, Pagel \& Tautvaisiene (1998) formally shifted data points for the LMC stars upwards by $0.2 \mathrm{dex}$. This was supposed to be a correction in order to adjust the data on LMC supergiants to those of Galactic supergiants. The latter stars show the mean negative value $[\mathrm{O} / \mathrm{Fe}]$ of about -0.2 dex based on the results of some works (e.g., Luck \& Lambert 1981, 1985).

Nevertheless, one important note has to be made. As it was shown by KA99, the oxygen abundances obtained for supergiant stars using the standard method can be significantly underestimated. Using the high quality multiphase spectra for famous Cepheid $\delta$ Cep KA99 obtained with a modified method the value $[\mathrm{O} / \mathrm{Fe}] \approx 0$. Moreover, the similar results were also recently obtained by us for the number of Galactic Cepheids (results are in preparation for publications). These results are in agreement with the value $[\mathrm{O} / \mathrm{Fe}]$ which is expected from the model of Galactic chemical evolution for $[\mathrm{Fe} / \mathrm{H}] \approx 0$ (see Wyse 1999).

Our new analysis provides both a lower metallicity and a higher $(\mathrm{O} / \mathrm{H})$ value than previous determinations, providing a value which is closer to what would be expected for this metallicity in the classical and global evolution of abundances in our Galaxy (Edvardsson et al. 1993; Wyse 1999): suggesting that the evolution of the LMC is slightly less different from the Galactic one than previously thought.

\subsection{Sodium}

High luminosity Galactic stars are known to have enhanced sodium lines, possibly a real enhancement of $\mathrm{Na}$ abundance due to $\mathrm{Ne}-\mathrm{Na}$ processed material mixed with the atmospheric gas. Recently, El Eid \& Champagne (1995) proposed a theory in which a sodium overproduction in supergiants increases with increasing stellar mass. Such an overproduction does not exceed 0.3-0.4 dex for 10-20 $M_{\odot}$. Our mean result for massive $\mathrm{F}$ supergiants indicates an abundance smaller than the LTE value, resulting in a very small enhancement $([\mathrm{Na} / \mathrm{Fe}]=+0.05)$, smaller than expected within the theory. This result is in agreement with LET98. The Galactic supergiants have also high $(\mathrm{Na} / \mathrm{Ca})$ ratios, and this peculiarity is not found here. Among several explanations, the difference with the Galactic supergiants, could be due to the suppression of sodium overproduction in metal deficient stars.

\subsection{General consequences for other elements}

The ratio $(\alpha$-elements $/ \mathrm{Fe})$ is less different from the predictions of the classical Galactic evolution. Magnesium was previously found without any enhancement, and even with a slight deficiency relative to iron, but in this work it is even lower (by a factor of 2). This overdeficiency is surprising, but let us note that such a NLTE overdeficiency of $\mathrm{Mg}$ is also found in the B-type supergiants of the SMC (Dufton et al. 2000), but not generally in A-type SMC supergiants (Venn 1999 and references therein).

Sulphur is one of the elements whose abundance can be also measured in $\mathrm{H}$ II regions. This element is not processed in the intermediate-to-high mass stars at their early evolutionary stages, therefore its abundance in the young supergiant atmospheres should reflect that of the ISM. Our mean $\mathrm{S}$ abundance obtained for a sample of $\mathrm{F}$ supergiants is $A(\mathrm{~S})=6.95 \pm 0.25$ (i.e. $[\mathrm{S} / \mathrm{H}]=-0.26$ ), while from Hil regions of LMC Dufour (1984) found $A(\mathrm{~S})=$ $6.85 \pm 0.11$, that within an error bar is practically the same value as ours.

It has to be noted that the mean iron abundance determined in the present study is $[\mathrm{Fe} / \mathrm{H}]=-0.40$, i.e. slightly lower than the value derived by HAS95, while LL92 and Korn et al. (2000) obtained the metallicity of $\mathrm{LMC}[\mathrm{Fe} / \mathrm{H}]=-0.36$ and -0.41 respectively. Both these estimates agree with our result. The relative abundances $[\mathrm{M} / \mathrm{H}]$ of iron-group elements are close to the iron content (see Fig. 1).

Even with an analysis made with different gravities, the abundances of the heavy elements (derived from the lines of ions) remain enhanced. This is clearly seen in Fig. 1. The (hs/ls) ratio is rather high and definitely non-solar.

\section{Conclusion}

The elemental abundances have been re-investigated in nine F supergiants of the Large Magellanic Cloud. The mean abundance of iron is found to be $[\mathrm{Fe} / \mathrm{H}]=-0.40$, slightly lower than previously adopted for LMC, but in accordance with the recent results obtained by Korn et al. (2000).

The NLTE calculations for carbon, oxygen and sodium, based on Kurucz's atmospheric models with an over-all metal deficiency, were carried out as described in Korotin et al. (1999). Importantly, the NLTE oxygen abundance is found higher and the carbon abundance lower than in previous work. The carbon abundance is in much better agreement with that of the LMC HII regions, oxygen remaining in fair agreement with $\mathrm{H}$ II regions. The $(\mathrm{C} / \mathrm{O})$ ratio is low, lower than solar and the $\mathrm{H}$ II ratio (a possible sign of the first dredge-up). The relative to iron abundance of oxygen $[\mathrm{O} / \mathrm{Fe}]=+0.01$ derived for nine $\mathrm{F}$ supergiants appears in excellent accordance with the prediction based on the smooth model of the LMC evolution.

We did not find a remarkable sodium overabundance in the LMC supergiants, at contrast to the Galactic 
supergiants that could be caused by inefficient sodium overproduction in $\mathrm{Ne}-\mathrm{Na}$ cycle in the metal deficient stars.

The $(\alpha / \mathrm{Fe})$ ratios are comparable to those that were obtained in previous analyses, with magnesium abundance being significantly lower in the new analysis. Sulphur abundances in supergiants show a good agreement with those measured in $\mathrm{H}$ II regions.

The iron-group elements follow the solar distribution of the $(\mathrm{M} / \mathrm{Fe})$ ratios. With a different method of gravity determination, for the elements heavier than iron-group ones, an enhancement remains, in agreement with previous studies.

For the LTE analysis, the non-standard approach, following KA99, leads to significantly larger $\log g$ values, and these larger gravities result in masses of supergiants of about $10-20 M_{\odot}$, that are in the excellent agreement with the evolutionary calculations. However, the evolutionary status of our program stars is not completely clear. Depending upon the used evolutionary tracks, our stars can be considered 1) either as those moving towards the red giant region for the first time, 2) or having already passed the red giant stage and experienced the first dredge-up, and now performing their blue loops.

Finally, one can note that an extension of this work to the SMC would be useful. The latter is planned to be presented in our next paper.

Acknowledgements. We thank Dr. O. R. Pols for having put in our disposal an electronic version of the evolutionary models. Our special thanks to Dr. R.-P. Kudritzki and N. Przybilla for the careful inspection of the manuscript and giving us many useful suggestions which significantly improved the initial version of the paper.

\section{References}

Allen, C. W. 1973, Astrophysical Quantities (Athlone Press, London)

Anders, E., \& Grevesse, N. 1989, Geochim. Cosmochim. Acta, 53, 197

Andrievsky, S. M., Korotin, S. A., Luck, R. E., \& Kostynchuk, L. Yu. 1999, A\&A, 350, 598

Biémont, E., Hibbert, A., Godefroid, M., Vaeck, N., \& Fawcett, B. C. 1991, ApJ, 375,818

Biémont, E., Hibbert, A., Godefroid, M., \& Vaeck, N. 1993, ApJ, 412, 431

Carlsson, M. 1986, Uppsala Obs. Rep., 33

Castelli, F. 1999, A\&A, 346, 564

Castelli, F., Gratton, R. G., \& Kurucz, R. L. 1997, A\&A, 318, 841

Dufour, R. J. 1984, Structure and evolution of the Magellanic Clouds, IAUS 108, ed. S. van den Bergh, \& K. S. de Boer, 353

Dufour, R. J., Shields, G. A., \& Talbot, R. J. Jr. 1982, ApJ, 252,461

Dufton, P. L., McErlean, N. D., Lennon, D. J., \& Ryans, R. S. I. 2000, A\&A, 353, 311
Edvardsson, B., Andersen, J., Gustafsson, B., et al. 1993, A\&A, 275,101

El Eid, M. F., \& Champagne, A. E. 1995, ApJ, 451, 298

Gratton, R. G., Caretta, E., Eriksson, K., \& Gustafsson, B. 1999, A\&A, 350, 955

Grevesse, N., \& Sauval, A. J. 1998, Space Sci. Rev., 85, 161

Grieve, G. R., \& Madore, B. F. 1986, ApJS, 62, 427

Hill, V., Andrievsky, S. M., \& Spite, M. 1995, A\&A, 293, 347 (HAS95)

Hirata, R., \& Horaguchi, T. 1994, Atomic spectral line list, CDS catalogue VI/69

Korn, A. J., Becker, S. R., Gummersbach, C. A., \& Wolf, B. 2000, A\&A, 353, 665

Korotin, S. A., Andrievsky, S. M., \& Luck, R. E. 1999, A\&A, 351, 168

Korotin, S. A., Andrievsky, S. M., \& Kostynchuk, L. Yu. 1999, Ap\&SS, 260, 531

Korotin, S. A., \& Mishenina, T. V. 1999, ARep, 43, 533

Kovtyukh, V. V., \& Andrievsky, S. M. 1999, A\&A, 351, 597 (KA99)

Kurt, C. M., \& Dufour, R. J. 1998, Rev. Mex. A. A. Ser. Conf., 7, 202

Kurucz, R. L. 1992, The Stellar Populations of Galaxies, ed. B. Barbuy, \& A. Renzini, IAU Symp., 149, 225

Kurucz, R. L., \& Bell, B. 1995, Atomic line data, CD-ROM, No. 23, SAO

Kurucz, R. L., Furenlid, I., Brault, I., \& Testerman, L. 1984, The Solar Flux Atlas from $296 \mathrm{~nm}$ to $1300 \mathrm{~nm}$, National Solar Observatory

Luck, R. E., \& Lambert, D. L. 1981, ApJ, 245, 1018

Luck, R. E., \& Lambert, D. L. 1985, ApJ, 298, 782

Luck, R. E., \& Lambert, D. L. 1992, ApJS, 79, 303 (LL92)

Luck, R. E., Moffett, T. J., Barnes III, T. G., \& Gieren, W. P. 1998, AJ, 115, 605 (LET98)

Maltby, P., Avrett, E. H., Carlsson, M., et al. 1986, ApJ, 306, 284

Mishenina, T. V., Korotin, S. A., Klochkova, V. G., \& Panchuk, V. E. 2000, A\&A, 353, 978

Pagel, B. E. J., Edmunds, M. G., Fosbury, R. A. E., \& Webster, B. L. 1978, MNRAS, 184, 569

Pagel, B. E. J., \& Tautvaisiene, G. 1998, MNRAS, 299, 535

Pilyugin, L. S. 1996, A\&A, 310, 751

Pols, O. R., Schroeder, K.-P., Hurley, J. R., Tout Ch. A., \& Eggleton, P. P. 1998, MNRAS, 298, 525

Van Regemorter, H. 1962, ApJ, 136, 906

Sahibullin, N. A. 1987, SvA, 31, 666

Sandage, A., Bell, R. A., \& Tripicco, M. J. 1999, ApJ, 522, 250

Schaller, G., Schaerer, D., Meynet, G., \& Maeder, A. 1992, A\&AS, 96, 269

Seaton, M. J. 1962, Proc. Phys. Soc., 79, 1105

Spite, F., Spite, M., \& François, P. 1989, A\&A, 210, 25

Steenbock, W., \& Holweger, H. 1984, A\&A, 130, 319

Takeda, Y., \& Takada-Hidai, M. 1994, PASJ, 46, 395

Thévenin, F., \& Idiart, T. P. 1999, ApJ, 521, 753

Venn, K. A. 1999, ApJ, 518, 405

Verner, D. A., Barthel, P. D., \& Tytler, D. 1994, A\&AS, 108, 287

Wyse, R. F. G. 1999, Ap\&SS, 267, 145

Yan, Y., Taylor, K. T., \& Seaton, M. J. 1987, J. Phys. B: At. Molec. Phys., 20, 6409 